\title{
No se observó relación entre el estatus tiroideo y la incidencia de eventos cardiovasculares
}

Cappola A y col. JAMA 2006; 295; 1033:1041

\section{Objetivo}

Determinar la relación entre el estatus tiroideo basal, la incidencia de fibrilación auricular, la enfermedad cardiovascular y la mortalidad en adultos mayores de 65 años que no recibían medicación tiroidea.

\section{Diseño}

Estudio de cohorte prospectivo.

\section{Lugar}

Base de datos del Cardiovascular Health Study (CHS), EE UU.

\section{Pacientes}

3233 individuos mayores de 65 años aleatorizados de cuatro comunidades de Estados Unidos, desde 1989-1990, pertenecientes al CHS. El seguimiento promedio fue de 13 años. Se excluyeron aquellos pacientes que estaban institucionalizados, que recibían tratamiento activo para cáncer o no estaban en condiciones de proveer consentimiento. A su vez tampoco fueron incluidos los pacientes que presentaban tirotoxicosis (definida o posible), enfermedades generales con alteraciones de la tirotrofina (TSH) de origen no tiroideo ni aquellos que utilizaban medicación tiroidea, corticoides $u$ otras drogas que afectaran la función tiroidea.

\section{Medición de resultado principal}

Se analizaron los sueros de 3233 pacientes y se midió TSH y T4L, determinándose cinco grupos (eutiroideo, hipotiroidismo subclínico, hipotiroidismo, hipertiroidismo subclínico e hipertiroidismo). En aquellos grupos en los que se encontró alguna alteración de la función tiroidea se comenzó con medicación. Se evaluó la incidencia de fibrilación auricular, enfermedad coronaria, accidente cerebrovascular y muerte por causa cardiovascular y por todas las causas en cada uno de los grupos.

Se evaluaron a su vez, como factores confundidores, las siguientes covariables: raza, colesterol total, HDL y LDL en ayunas, triglicéri- dos, hipertensión arterial (HTA) (y/o uso de drogas antihipertensivas) diabetes (o utilización insulina y/o hipoglucemiantes orales) glucemia en ayunas alterada mayor a 100 y proteína $C$ reactiva.

\section{Resultados principales}

No hubo diferencias entre las distintas categorías de estatus tiroideo y los eventos evaluados al inicio del estudio. En la tabla 1 se muestran la relación entre el estatus tiroideo y la incidencia de eventos a los 13 años de seguimiento, expresada como riesgo de eventos tomando en comparación el grupo eutiroideo.

\section{Tabla 1. Eventos cardiovasculares y estatus tiroideo.}

\begin{tabular}{|c|c|c|c|c|c|}
\hline \multicolumn{6}{|c|}{ Hazard ratio (C 95\%) } \\
\hline & \begin{tabular}{|l|} 
Fibrilación \\
auricular(*)
\end{tabular} & \begin{tabular}{|l|} 
Enfermedad \\
coronaria(**)
\end{tabular} & $\begin{array}{l}\text { Accidente cerebro } \\
\text { vascular (**) }\end{array}$ & $\begin{array}{c}\text { Muerte } \\
\text { carcliovascular(**) }\end{array}$ & Muerte(**) \\
\hline Eutiroideo(n=2639) & 1 & 1 & 1 & 1 & 1 \\
\hline Hipotirodilismo subclinico(n=496) 1 & $1,11(0,92-1,34)$ & $1,07(0,90-1,28)$ & $1,01(0,79-1,29)$ & $1,16(0,92-1,46)$ & $1,14(0,98-1,32)$ \\
\hline Hipotiroidismo(n=18) & $0,88(0,48-1,63)$ & $0,93(0,57-1,53)$ & $0,86(0,42-1,76)$ & $0,97(0,49-1,92)$ & $1,43(0,95-2,14)$ \\
\hline Hipertirodidismo subclínico(n=7) 2 & $2,18(1,42-3,33)$ & $1,04(0,64-1,69)$ & $0,70(0,31-1,57)$ & $0,94(0,49-1,83)$ & $1,08(0,72-1,62)$ \\
\hline
\end{tabular}

(*) Ajustado por edad, sexo, enfermedad cardiovascular y uso de medicación tiroidea durante el seguimiento.

$\left({ }^{* *}\right)$ Ajustado por edad, sexo, enfermedad cardiovascular, fibrilación auricular, uso de medicación tiroidea durante el seguimiento, raza, tabaquismo, diabetes, LDL, HTA, índice de masa corporal y proteina $\mathrm{C}$ reactiva.

\section{Conclusiones}

Los datos globales del estudio no muestran relación entre el status tiroideo y la incidencia de eventos en lo que respecta a ACV, enfermedad coronaria, mortalidad cardiovascular y mortalidad por todas las causas. Sí se observó en el grupo de pacientes con hipertiroidismo subclínico una mayor incidencia de fibrilación auricular.

Fuente de financiamiento: American Heart Association Grant, National Heart, Lung and Blood Institute y National Institute on Aging.

\section{Comentario}

La enfermedad tiroidea es oligosintomática en los adultos mayores de 65 años, y en los límites del hipo y del hipertiroidismo subclínicos puede pasar inadvertida'.

Si bien hay grupos de trabajo que recomiendan el rastreo sistemático de la disfunción tiroidea, no hay consenso generalizado sobre el tema'.

Una de las fortalezas del estudio es el seguimiento durante 12 años, lo cual contribuye a la validez del estudio.

Este trabajo además, concuerda con el Framinghan Heart Study, donde en personas mayores de 60 años también se observó mayor incidencia de fibrilación auricular, aunque el desarrollo de ésta fue analizado en relación a la concentración de TSH y no se analizaron por separado aquellos pacientes que presentaban hipertiroidismo de los que padecían hipertiroidismo subclínico.

Por otro lado la información es clara en cuanto al beneficio del tratamiento del hipertiroidismo subclínico en lo que respecta a la fibrilación auricular y progresión a hipertiroidismo en algunos subgrupos especiales, como los mayores de 65 años$^{2}$.
Es importante tener en cuenta la selección cuidadosa que hubo de los pacientes en esta cohorte, ya que en el contexto de nuestro consultorio los pacientes se presentan con enfermedades generales no tiroideas $y / 0$ tratamientos instituidos, los cuales pueden afectar el valor de TSH.

\section{Conclusiones del comentador}

Si bien la población con hipertiroidismo subclínico tiene mayor riesgo de evolucionar con fibrilación auricular en su historia natural, deberíamos realizar reastreo en 2500 adultos mayores para hallar un caso de fibrilación auricular asociado a hipertiroidismo subclínico. Como vemos, el costo y la utilización de recursos se incrementan de manera exponencial sin obtener resultados contundentes, ya que el hipertiroidismo subclínico no muestra aumento de la mortalidad respecto de la población eutiroidea.

Andrea Pechín [ Médica Especialista en Medicina Familiar. Servicio de Endocrinología del Hospital Italiano de Buenos Aires. ]

Recibido el 15/8/06 y aceptado el 25/9/06.

Pechin A. No se observó relación entre el estatus tiroideo y la incidencia de eventos cardiovasculares. Evid. actual. práct. ambul.;9(5):141. Sep-Oct. 2006. Comentado de: Cappola A. Fried L. Arnold A. Thyroid Status, Cardiovascular Risk, and Mortality in Older Adults. JAMA 2006; 295:1033-1041. PMID: 16507804.

\section{Referencias}

1. Ross D. Euthyroid hyperthyroxinemia and hypothyroxinemia. Surks M lodide and thyroid function. Ross D. Laboratory assessment of thyroid function. En 2006, UpToDate. Disponible en URL: www.uptodate.com. Ultimo acceso: 13.22005

2. Surks MI, Ortiz E, Daniels GH et al. Subclinical thyroid disease: scientific review and guidelines for diagnosis and management. JAMA 2004 Jan 14; 291(2):228-38. 